\title{
The influence of promiscuous metals on metalloprotein structure: Complementary techniques to separate the good, the bad, and the ugly.
}

Edward H. Snell ${ }^{1,2}$, M. Elizabeth Snell ${ }^{1}$, Oliver B. Zeldin ${ }^{3}$, Geoff W. Grime ${ }^{4}$, and Elspeth F. Garman $^{3}$

1. Hauptman-Woodward Medical Research Institute, 700 Ellicott St., Buffalo, NY 14203, USA, 2. SUNY Buffalo Department of Structural Biology, Hauptman-Woodward Medical Research Institute, 700 Ellicott St., Buffalo, NY 14203, USA, 3. Department of Biochemistry, University of Oxford, South Parks Road, Oxford OX1 3QU, UK. 4. Ion Beam Centre, Advanced Technology Institute, University of Surrey, Guildford GU2 7XH, U.K.

At least one third of all proteins are metalloproteins, with one or more metals playing a structural or catalytic role. The identity and stoichiometry of these inorganic ligands are vital pieces of biophysical data for characterizing proteins, but there is currently no widely accepted standard assay for this analysis. In cases where the structure is known, circumstantial evidence from the atomic resolution structures is utilised, but this has been shown to be unreliable, particularly at low resolutions. In particular, when X-ray crystallography is employed, the choice of metal made at the refinement stage will affect the restraints, biasing the final structure towards the putative metal choice. When no structural information are available, bioinformatic approaches can be used, but direct experimental measurement is not part of routine characterization protocols.

Metals are promiscuous during crystallization. In many cases the metal associated with a structural model may not be the native metal, since one contained in the crystallization cocktail may have competed with it and won. While successful crystallization conditions can indicate that this may be probable, no technique that studies the crystal alone can identify these cases or confirm which metal was originally associated with the protein.

We show that a combination of particle induced X-ray emission (PIXE) and comprehensive analysis of crystallization screening outcome can identify erroneous metals, indicate promiscuity in the crystallization stage, and improve the quality of the resultant structural model and the information that can be obtained from it. By combining two high-throughput approaches, our method provides a tool that could conceivably find routine use in the field. 